\title{
Evaluation of an Educational Program for Clinical Pharmacists to Conduct Standardized Assessments for Medication-Induced Movement-Related Disorders
}

\author{
Alessandra Spadaro, Jamie Kellar, Gary Remington, Beth Sproule, Mayce Al-Sukhni, \\ and Albert Chaiet
}

\section{INTRODUCTION}

$\mathrm{M}$ ovement disorders and tardive dyskinesia are common adverse effects of first-generation antipsychotic medications. Although the atypical agents are less likely to result in movement disorders, they are not entirely devoid of this risk. Movement disorders such as akathisia continue to be associated with newer antipsychotics like risperidone and aripiprazole, ${ }^{1,2}$ and all currently available antipsychotics carry a risk of tardive dyskinesia. ${ }^{3,4}$ In addition, the anticipated benefits of atypical antipsychotics have been tempered, and they come with their own unique set of adverse effects, factors that together have led to a re-evaluation of the use of conventional antipsychotics. ${ }^{5}$ Antipsychotic-induced movement disorders and tardive dyskinesia are often underrecognized in clinical practice, with potentially damaging implications for patient care. ${ }^{6}$ Despite the availability of validated rating scales, evidence of their use remains scarce. Studies have revealed a lack of documentation (both quantity and quality) of movement disorder assessment, as well as challenges associated with accurate detection and management of these adverse effects. ${ }^{7-9}$

Distinguishing one movement disorder from another continues to be challenging and requires careful evaluation by experienced raters. ${ }^{10}$ Pharmacists are well positioned to fill this role. Pharmacists build their practice according to a pharmaceutical care model in which they are held accountable for providing rational drug therapy with the goal of optimizing patient outcomes. ${ }^{11}$ This goal is achieved by regularly monitoring patient-specific medical data, evaluating the management of medication, and providing pharmaceutical care for the purpose of identifying and resolving drug therapy problems. ${ }^{12,13}$ Numerous published reports have shown that the provision of clinical pharmacy services, including medication interventions, has resulted in better patient care, shorter hospital stays, and health care cost savings. ${ }^{14}$ The success of pharmacists in managing diabetes mellitus, lipid abnormalities, anticoagulation, and complex HIV drug regimens has been well documented. ${ }^{15,16}$ Despite these expanded roles in some settings, pharmacists have been found to represent an underutilized health care resource. ${ }^{17}$

In the field of mental health, research related to the provision of specific pharmaceutical care services is limited. As such, there is a unique opportunity to study the impact of training pharmacists to assess movement disorders. Pharmacists are in an excellent position to conduct such assessments, as they have regular contact with patients and are experts in medication management, which includes the evaluation and management of adverse effects. At the authors' clinical site, formal training for such assessments is currently unavailable to pharmacists who routinely work with patients receiving antipsychotic therapy. The value of formal clinician training to better identify antipsychotic-induced movement disorders is emphasized in the literature, including the benefits of having trained pharmacists screen patients for the purpose of identifying and managing these adverse effects. ${ }^{18-20}$

In this study, investigators developed a new program to train clinical pharmacists to assess medication-related movement disorders. This research functioned as a pilot study, with only a small number of participants, with the intention to provide broader implementation of the program if successful.

\section{METHODS}

\section{Study Design}

The study took place at the Centre for Addiction and Mental Health, in Toronto, Ontario, which is Canada's largest psychiatric teaching hospital. Ten pharmacists were trained to assess movement disorders in patients receiving antipsychotic therapy. Pharmacists with previous formal movement-disorder training were excluded. Participants were trained to use 3 standardized 
rating scales: the modified Simpson Angus Scale (MSAS) for parkinsonian symptoms, the Barnes Akathisia Rating Scale (BARS) for akathisia, and the Abnormal Involuntary Movement Scale (AIMS) for tardive dyskinesia. ${ }^{21}$ Because of their established validity and reliability, the AIMS, BARS, and Simpson Angus Scale are the rating scales most extensively used in clinical trials. ${ }^{10}$ The original developers of the Simpson Angus Scale created the MSAS, but have not published any research on the modified version. Nonetheless, the MSAS was used for the current study, as it (rather than the original scale) is used in recent training videos. The pilot program consisted of 2 training sessions presented on 2 study days and involved a combination of videotaped and live patient assessments. The ability of each participant to correctly identify a movement disorder was evaluated before and after each training session. This study was approved by the Centre for Addiction and Mental Health Research Ethics Board.

\section{Study Day 1: Video Training}

Baseline Assessment: Before training, all study participants completed a questionnaire to determine their knowledge of, comfort with, and confidence in the area of movement disorders. An information sheet on movement disorders was circulated and discussed by one of the investigators (A.S.), who facilitated the session. Participants were given time to familiarize themselves with the movement disorder scales before performing any assessments. Participants then independently assessed and scored 3 patients from videos included in a training DVD produced by the College of Psychiatric and Neurologic Pharmacists ${ }^{22}$ : one patient was assessed with the MSAS, a second patient with the BARS, and a third patient with the AIMS.

Video Training: Results were collected and the correct scoring for the baseline video assessments (defined by the video's experts) was shared with the participants, for comparison with their own assessments. Several additional video clips from the same source were then shown, which included an introduction to each of the scales, information about assessing the severity of a movement disorder, and techniques to conduct physical examinations. The patient examinations used in the baseline assessment were re-played, which included the video experts' scoring rationale for each of the assessments.

Assessment after Video Training: Using the same DVD, participants then independently scored 3 new patients in a similar manner. Results were collected, and the correct scoring rationale was shown via video after each assessment. Investigators compared the percent absolute agreement among participants with the expert rater on each item of each scale.

Scores obtained before and after the video training were compared to determine whether there had been any improvement.

\section{Study Day 2: Live Patient Interviews and Discussion}

A psychiatrist (G.R.), who is an expert in the fields of psychiatry and antipsychotic-related movement disorders, facilitated this session. To begin, participants were given time to ask questions about movement disorders. Three patients who had previously participated in clinician-training activities related to movement disorders volunteered their time for this session. The facilitator examined 2 of these patients, verbally working through all 3 scales during each examination, while study participants followed along with their own scoring. After each examination, participants discussed the scoring results and compared their own assessments with that of the expert rater. These 2 examinations were considered practice for the participants, and the results were not included in the analysis. The facilitator then examined the third patient, providing verbal commentary without referring to specific aspects of the scales, while participants scored the patient on each of the 3 rating scales. After results were collected, the participants were given time to discuss the correct scoring with the psychiatrist. Scores for each item of each scale were compared with expert rater responses for this patient, and the percent absolute agreement was determined. Participants then completed another questionnaire similar to that completed on study day 1 .

\section{Statistical Analysis}

Following consultation with a biostatistical service, it was decided to use percent agreement to evaluate participants' performance with the rating scales. One-tailed, paired $t$ tests $(\alpha<0.05)$ were computed to compare overall mean values for each scale between experts and participants. In addition, individual scores for each item were summed to generate a total score per patient, which was used to categorize overall severity. The total score was compared with the overall severity score assigned by the expert rater (deemed the "true severity"). Questionnaire responses were analyzed descriptively to provide insight into participants' overall impressions of the training program.

\section{RESULTS}

All 10 participants completed study day 1 . One participant was unable to attend study day 2 because of illness.

\section{Modified Simpson Angus Scale (MSAS)}

For the MSAS, the average percent absolute agreement ( \pm standard deviation) with the expert rater did not differ significantly before and after training on study day $1(66 \% \pm 22 \%$ versus $61 \% \pm 34 \%$, respectively; $p=0.37$ ) (Table 1 ). Agreement on overall severity increased from $80 \%$ before training to $100 \%$ after training on study day 1 . The participants tended to overestimate the severity of each item both before and after training on study day 1 .

Agreement for post-training scoring on study day 2 was greater for all items where an increase was possible. Average agreement increased to $99 \% \pm 3 \%(p<0.001)$ relative to baseline assessments. Agreement between participants and the expert rater was consistently above $80 \%$ on the more objective items of the MSAS, such as glabella tap, tremor, salivation, and akathisia, throughout all sessions (Table 1). 
This single copy is for your personal, non-commercial use only.

For permission to reprint multiple copies or to order presentation-ready copies for distribution, contact CJHP at cjhpedit@cshp.ca

Table 1. Absolute Agreement with Expert Rater for the Modified Simpson Angus Scale

Timeframe; \% Agreement with Expert Rater*

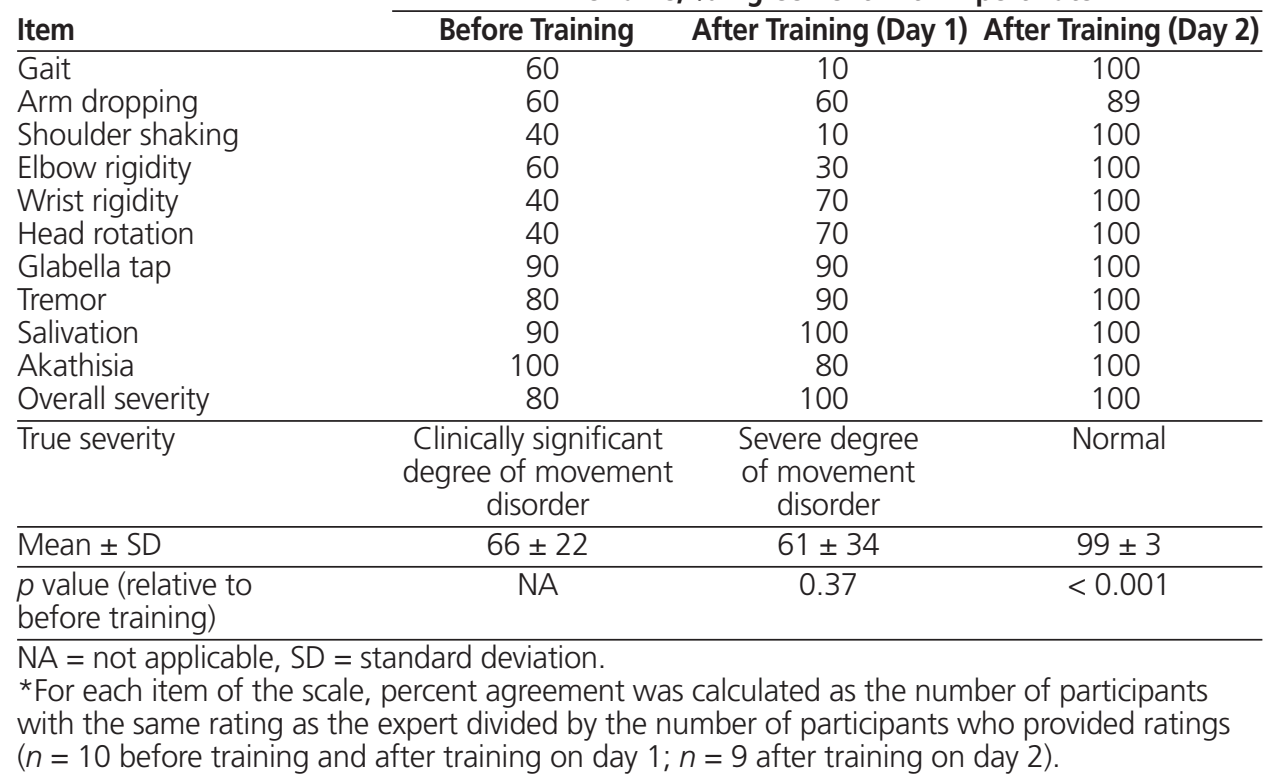

Table 2. Absolute Agreement with Expert Rater for the Barnes Akathisia Rating Scale

Timeframe; \% Agreement with Expert Rater*

\begin{tabular}{lccc} 
& Before Training & After Training (Day 1) & After Training (Day 2) \\
\cline { 2 - 4 } Objective & 80 & 10 & 100 \\
Subjective awareness & 40 & 100 & 100 \\
Subjective distress & 20 & 100 & 100 \\
Global assessment & 40 & 70 & 100 \\
\hline True severity & Marked akathisia & Absent & Absent \\
& & (pseudoakathisia) & (no akathisia) \\
\hline Mean \pm SD & $45 \pm 25$ & 0.25 & $100 \pm 0$ \\
\hline p value (relative to & NA & & 0.01 \\
before training) & & & \\
NA * not applicable, SD = standard deviation. & \\
*For each item of the scale, percent agreement was calculated as the number of participants \\
with the same rating as the expert divided by the number of participants who provided ratings \\
( $n=10$ before training and after training on day $1 ; n=9$ after training on day 2).
\end{tabular}

\section{Barnes Akathisia Rating Scale (BARS)}

Participants' agreement with the expert rater, averaged over all items of the BARS, was $45 \% \pm 25 \%$ at baseline, $70 \% \pm 42 \%$ after video training on study day 1 ( $p=0.25$ relative to baseline), and $100 \%$ after live training on study day $2(p=0.01$ relative to baseline) (Table 2). Before training, the participants were more accurate in scoring objective assessments of akathisia than subjective measures (e.g., restlessness), whereas by the end of study day 1 , the opposite was observed. The participants tended to underestimate the global severity rating for the patient at baseline, whereas they overestimated severity after training on study day 1 .

\section{Abnormal Involuntary Movement Scale (AIMS)}

Participants' agreement with the expert rater, averaged over all items of the AIMS, was 33\% $\pm 22 \%$ before training, $50 \%$ $\pm 30 \%$ after video training on study day 1 ( $p=0.07$ relative to baseline), and $58 \% \pm 37 \%$ after live training on study day 2 $(p=0.08$ relative to baseline) (Table 3 ). Agreement for the awareness and distress item remained consistently low (10\%), even after training. The participants tended to overestimate the overall severity of the patient's movement disorder at baseline, whereas the opposite occurred after training on study day 1 . The same proportion of participants over- and under-estimated the severity of the patient scored on study day 2 . 
This single copy is for your personal, non-commercial use only.

For permission to reprint multiple copies or to order presentation-ready copies for distribution, contact CJHP at cjhpedit@cshp.ca

\section{Table 3. Absolute Agreement with Expert Rater for the Abnormal Involuntary Movement Scale}

Timeframe; \% Agreement with Expert Rater*

\begin{tabular}{|c|c|c|c|}
\hline Item & Before Training & After Training (Day 1) & After Training (Day 2) \\
\hline Facial muscles & 20 & 60 & 33 \\
\hline Lips/perioral area & 20 & 80 & 100 \\
\hline Jaw & 60 & 40 & 89 \\
\hline Tongue & 10 & 60 & 78 \\
\hline Upper extremities & 40 & 60 & 0 \\
\hline Lower extremities & 70 & 100 & 0 \\
\hline Trunk movements & 10 & 40 & 100 \\
\hline Overall severity & 40 & 50 & 56 \\
\hline Incapacitation & 50 & 0 & 67 \\
\hline Patient awareness and distress & 10 & 10 & 56 \\
\hline True severity & Minimal & Moderate & Mild \\
\hline Mean \pm SD & $33 \pm 22$ & $50 \pm 30$ & $58 \pm 37$ \\
\hline $\begin{array}{l}p \text { value (relative to } \\
\text { before training) }\end{array}$ & NA & 0.07 & 0.08 \\
\hline
\end{tabular}

\section{Questionnaires}

\section{Physical Assessments}

Nine $(90 \%)$ of the 10 participants reported no previous training in the performance of physical examinations and stated that they were either "not at all" or "somewhat" comfortable in performing physical assessments. Participants reported similar levels of confidence in accurately performing physical examinations.

\section{Assessment of Movement Disorders}

Nine (90\%) of the 10 participants reported encountering patients on antipsychotic therapy "very often" in their current practice. Before training, reported knowledge in the area of movement disorders ranged from limited to very knowledgeable. Of the 9 participants who completed the training program, 3 (33\%) noted that their knowledge remained consistently moderate before and after training, whereas 5 (56\%) reported an increase in knowledge (Figure 1A).

When asked to rate their comfort in the use of rating scales to assess patients for movement disorders, all participants reported feeling either "not at all" or "somewhat" comfortable before training. At the end of study day 2, all participants who completed the program reported an increase in comfort with performing assessments (Figure 1B).

At baseline, 9 (90\%) of the 10 participants reported feeling "not at all" or "somewhat" confident in their ability to accurately assess a patient for a movement disorder. A shift was observed after completion of the training program, whereby 2 (22\%) of the 9 fully trained participants felt "very" confident in their ability to accurately assess patients for movement disorders; none of the participants reported this confidence level before training (Figure 1C).

\section{Impressions of the Training Program}

Participants were asked to comment on the usefulness of the video and live patient training sessions in enhancing their understanding of examination techniques and the use of movement disorder rating scales. Four (44\%) of the 9 participants who completed the training found the video "somewhat" helpful for understanding examination techniques, whereas fewer found it "moderately" or "very" helpful. In contrast, 8 (89\%) of the participants found the live patient session either "very" or "extremely" helpful. Five (56\%) of the participants felt that the video was "moderately" helpful in enhancing their understanding of the use of rating scales. The same number of participants felt that live patient training was "extremely" helpful. Participants expressed diverse opinions when asked to comment on potential barriers to assessing movement disorders.

\section{DISCUSSION}

An overall improvement was observed in the ability of pharmacists participating in this pilot study to use rating scales to correctly identify movement disorders, as indicated by an increase in percent agreement with an expert rater after completion of the training program (Figure 2).

At baseline, participants scored more accurately on the MSAS than on the other 2 scales. The MSAS contains a mixture of subjective (e.g., rigidity, arm dropping) and objective (e.g., glabella tap, salivation, objective akathisia) measures of parkinsonism. Participants tended to agree with the expert rater on these arguably more objective measures, which may have inflated the average agreement by serving as a proxy for the overall score. The more subjective measures of rigidity may have been difficult to score from the video, which may have contributed to lower percent agreement for these items of the scale throughout study 


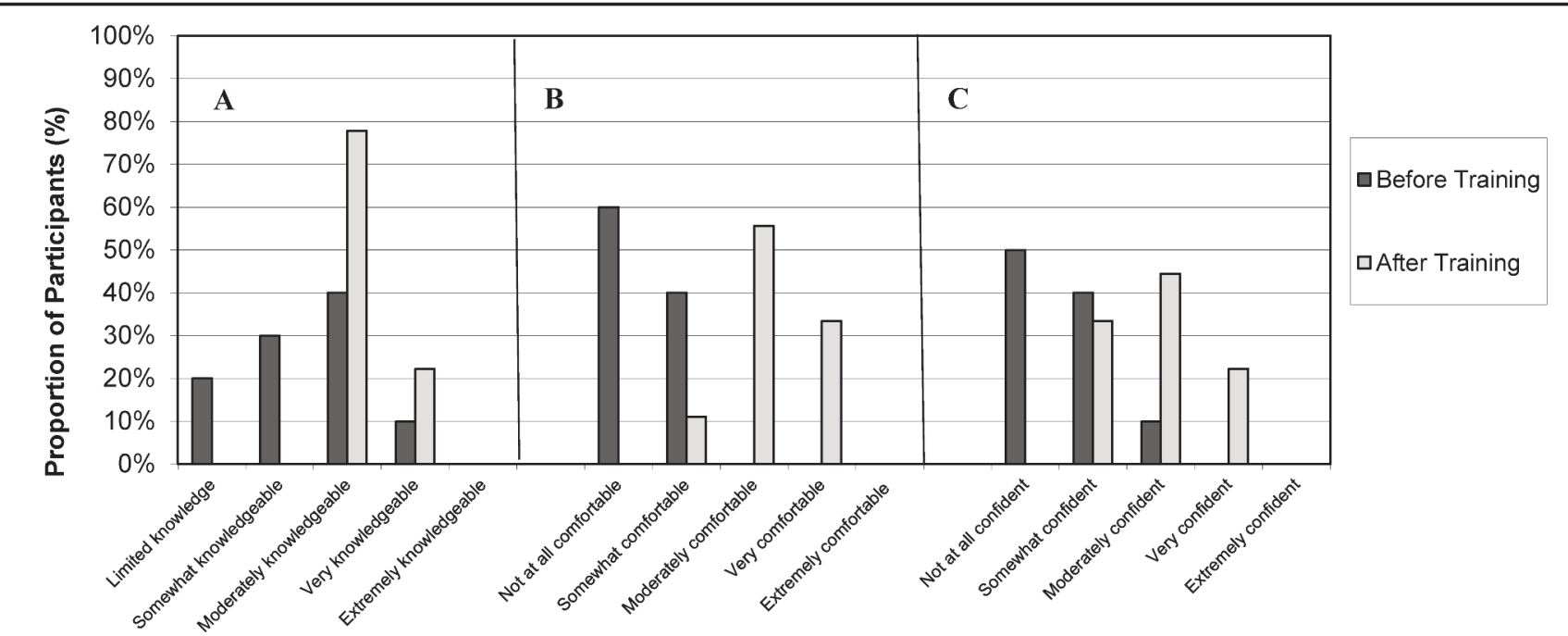

Figure 1. Participants' self-reported knowledge of (A), comfort with (B), and confidence in (C) the assessment of movement disorders ( $n=10$ before training, $n=9$ after training).

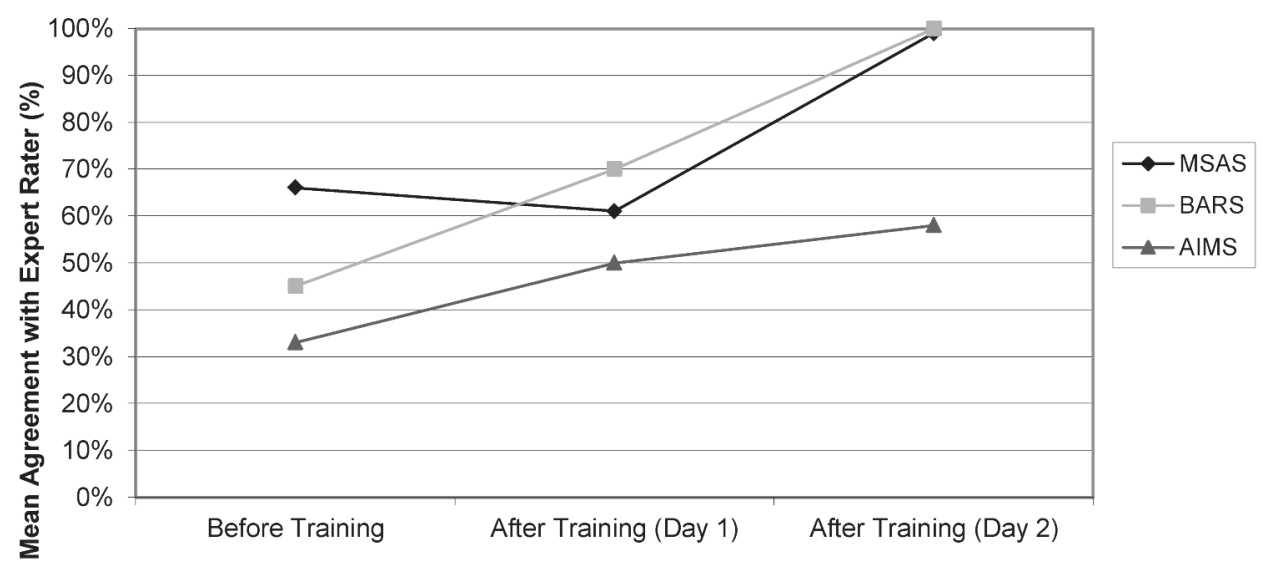

Figure 2. Absolute agreement for each scale before and after training. MSAS = Modified Simpson Angus Scale, BARS = Barnes Akathisia Rating Scale, AIMS = Abnormal Involuntary Movement Scale.

day 1 . On study day 2, participants observed examination of a real patient by the psychiatrist facilitating the session. Compared with the 2 video-based assessments on study day 1, an improvement in scoring was seen across all items of the MSAS, resulting in a statistically significant increase in overall agreement. Because of time constraints in the training session, participants did not have a chance to physically examine the live patient themselves. The observed increase in agreement may therefore have been biased by the examiner's verbal descriptions for measures of rigidity. This particular patient's severity of movement disorder was "normal" (as defined by the MSAS), and it may have been easier for participants to score this patient than individuals exhibiting true signs or symptoms of drug-induced parkinsonism.
At baseline, participants agreed with the rater to a greater extent for objective signs of akathisia than for subjective ratings of restlessness and distress on the BARS. During the baseline assessment, the examiner in the video suggested that the patient's expression of a desire to stop his medication indicated that he was severely distressed by his symptoms. There was some disagreement with this view among participants, who stated that the patient's affect did not appear consistent with someone who was particularly distressed by these symptoms. The "patient" in the video was actually a standardized actor, whose distress may have appeared more subtle than would be the case for a real patient. At the end of training on study day 1, participants' ratings of the subjective symptoms of akathisia (both items) were 
in $100 \%$ agreement with those of the expert rater. The subjective symptoms of akathisia may have been relatively easy to rate, as the patient expressed no awareness of and thus no distress related to restlessness. In contrast, participants scored worse on objective ratings of akathisia for this patient, with $5(50 \%)$ of the participants underestimating and $4(40 \%)$ overestimating the patient's rubbing of her clothes as an objective sign of akathisia. This result shows that more experience may be required to differentiate among levels of severity for akathisia. Upon completion of the training program on study day 2 , there was no disagreement between participants and the expert rater on any items of the BARS, resulting in a statistically significant overall increase in agreement relative to baseline. The patient assessed on study day 2 did not exhibit any objective signs of akathisia and did not express any internal feelings of restlessness. It may have been easier for participants to assess the absence of akathisia in this patient than it would have been to assess the presence of more severe forms of akathisia. By the end of study day 2, participants' performance on this scale was identical with their performance on the MSAS.

Participants struggled the most with the AIMS assessment, as evidenced by the consistently low average percent agreement at all 3 time points. This result was not surprising, given the greater complexity and detail required to complete this scale. At baseline, participants agreed with the expert rater to a greater extent on items where the patient's signs were absent or normal, further suggesting that an absence of symptoms may be clearer to pharmacists than a situation in which they must define the severity of observable signs of a movement disorder. Although improvement in most items of the AIMS was observed after training on study day 1 , some inconsistency in rating was still present for the items of patient awareness/distress and incapacitation. These items are arguably more subjective, as patients shown in the video were not explicitly asked to rate their levels of distress or incapacitation. On study day 2, however, the examiner explicitly asked the live patient to rate distress and incapacitation on the same ordinal scale as used for other items in the AIMS, and agreement on scoring of distress and incapacitation improved. After completion of training on study day 2, agreement was greater than pre-training and post-training agreement on study day 1 for all but 3 items on the AIMS. The examiner reported that this patient's movements had typically been more severe during previous assessments and that these 3 items (facial muscles, upper extremities, and lower extremities) were "difficult to notice", as they appeared milder in severity and occurred at the end of the interaction, as the patient was leaving the room. This situation highlights the need to assess a patient for movement disorders more than once in practice to establish an appropriate baseline rating, as symptom presentation may vary from one assessment to another. Participants were once again accurate in scoring the absence of movements during this session.

In general, participants tended to overestimate the overall severity of the patients' movement disorders at baseline. This result is consistent with that of a previous study in which untrained raters tended to overestimate the degree of tardive dyskinesia, while the opposite occurred after training. ${ }^{19}$ Overestimation of overall severity was not observed for pretraining scores on the BARS scale, for which the patient being scored had marked akathisia and there was arguably less opportunity for untrained participants to overestimate severity.

Participants found the live training session more beneficial than the video session in enhancing their understanding of examination techniques and the use of rating scales. This finding is consistent with the statistically significant improvement in agreement observed for the MSAS and BARS after completion of training on study day 2. Subjectively, participants desired discussion with a knowledgeable practitioner in the field of movement disorders and felt that live patient interaction was more valuable and realistic than observation of standardized patients on video. Participants also expressed the desire to rate more patients during their training, suggesting that the observation of 3 live patients was insufficient to grasp the entire range of severity of movement disorders.

Most participants reported no previous training in performing physical examinations. This lack of formal training may have been associated with the lack of comfort with and confidence in performing any type of physical assessment that participants reported at baseline. All but one participant reported a moderate or lower level of knowledge of movement disorders before training. By the end of the training program, participants reported feeling more knowledgeable, more comfortable, and more confident in their ability to assess a movement disorder. Although this type of study may have attracted pharmacists who felt less knowledgeable about the topic, these results suggest that formal training of pharmacists in the assessment of movement disorders is effective and warranted.

The results reported here are consistent with those of previous studies involving other health care professions. In a similarly designed study using lectures, videotapes, a training manual, and live patient exams, Kalachnik and others ${ }^{19}$ concluded that a wide variety of professionals, including physicians, nurses, and pharmacists, could be trained to reliably assess patients for tardive dyskinesia. Dixon and others ${ }^{20}$ compared standard psychopharmacology training of medical residents with more intensive training that focused primarily on extrapyramidal symptoms. The authors concluded that brief and focused training on extrapyramidal symptoms promoted improvements in the management of neuroleptic prescriptions and in the ability to diagnose these symptoms. ${ }^{20}$

Several limitations prevented the use of more statistically rigorous measures of inter-rater reliability, such as the kappa statistic or intra-class correlations. These limitations included the mixture of ordinal and nominal items on the rating scales, the small number of available patients, and the combination of videotaped and live patient examinations. Percent agreement was therefore the method of choice, although it does not take chance 
into account. The inflexibility of video examinations forced participants to rely on the examiner's accounts of the rigidity that is characteristic of drug-induced parkinsonism. This limitation likely biased participants' scoring, which might have differed to a greater extent from that of the expert if participants had been able to physically assess the patient for rigidity. The small number of patients assessed by participants was likely insufficient to allow the authors to fully gauge the program's effectiveness. The small number of patients also made results difficult to interpret, especially given that some of the patients did not exhibit signs or symptoms of a movement disorder. Although the inclusion of patients with no movement disorder in this pilot program might have reduced inter-rater variability, it is noteworthy that the participants did not demonstrate full agreement with the expert rater even for these patients. Ideally, pharmacists should perform assessments on numerous patients to learn to more accurately distinguish the severity of different movement disorders. However, increasing the number of patients assessed during training may be challenging outside of large psychiatric institutions, where this particular patient population may be sparse. This situation emphasizes some of the logistical benefits of videotaped over live patient training programs. The questionnaires completed by participants were not validated or standardized. Thus, differences between participants' and researchers' definitions of knowledge, comfort, and confidence may have biased the interpretation of the results.

\section{CONCLUSIONS}

Pharmacists participating in this study demonstrated an increased ability to accurately assess patients for movement disorders after completing the training program. Evaluation of the program indicated that pharmacists can be trained to use the MSAS, BARS, and AIMS, as evidenced by improvement in rater agreement and the increased knowledge, comfort, and confidence reported by participants in utilizing these scales. Study investigators and participants agreed that having the opportunity to independently score more patients would be a valuable enhancement to the training program and would reduce bias. Pharmacists may also benefit from more in-depth discussion or didactic lectures on the topic of movement disorders, as well as the use of rating scales in a practice setting. Training pharmacists to assess drug-induced movement disorders has the potential to enhance early detection and improve ongoing monitoring in patients.

\section{References}

1. Abilify product monograph. Montreal (QC): Bristol-Myers Squibb Canada; 2013 May 27

2. Risperdal product monograph. Toronto (ON): Janssen Inc; 2014 Nov 6.

3. Remington $\mathrm{G}$. Tardive dyskinesia: eliminated, forgotten, or overshadowed? Curr Opin Psychiatry. 2007;20(2):131-7.

4. Correll C, Schenk E. Tardive dyskinesia and new antipsychotics. Curr Opin Psychiatry. 2008;21(2):151-6.

5. Kane, J. Tardive dyskinesia circa 2006 [editorial]. Am J Psychiatry. 2006;163(8):1316-8.

6. Weiden P. Antipsychotic-induced movement disorders - forgotten but not gone [editoral]. Acta Psychiatr Scand. 2008;117(6):401-2.
7. Weiden P, Mann JJ, Gretchen H, Mattson M, Frances A. Clinical nonrecognition of neuroleptic-induced movement disorders: a cautionary study. Am J Psychiatry. 1987;144(9):1148-53.

8. Hansen TE, Brown WL, Weigel RM, Casey DE. Underrecognition of tardive dyskinesia and drug-induced parkinsonism by psychiatric residents. Gen Hosp Psychiatry. 1992;14(5):3404.

9. Cortese L, Jog M, McAuley TJ, Kotteda V, Costa G. Assessing and monitoring antipsychotic-induced movement disorders in hospitalized patients: a cautionary study. Can J Psychiatry. 2004;49(1):31-6.

10. Gervin M, Barnes TRE. Assessment of drug-related movement disorders in schizophrenia. Adv Psychiatr Treat. 2000;6(5):332-41.

11. Cipolle RJ, Strand L, Morley P, editors. Pharmaceutical care practice: the patient-centered approach to medication management services. 3rd ed. Columbus (OH): McGraw-Hill Education; 2012

12. Burke JM, Millar WA, Spencer AP, Crank CW, Adkins L, Bertch KE, et al.; American College of Clinical Pharmacy. Clinical pharmacist competencies. Pharmacotherapy. 2008;28(6):806-15.

13. Pottie K, Haydt S, Farrell B, Kennie N, Sellors C, Martin C, et al. Pharmacist's identity development within multidisciplinary primary health care teams in Ontario; qualitative results from the IMPACT project. Res Soc Admin Pharm. 2009;5(4):319-26.

14. Virani A, Crown N. The impact of a clinical pharmacist on patient and economic outcomes in a child and adolescent mental health unit. Can J Hosp Pharm. 2003;56(3):158-62.

15. Smith M. Pharmacists' role in improving diabetes medication management. J Diabetes Sci Technol. 2009;3(1):175-9.

16. Specialty pharmacists boost the care and compliance of HIV-infected patients. Dis Manag Advis. 2007;13(3):29-31.

17. Smith M. Pharmacists and the primary care workforce. Ann Pharmacother. 2012;46(11):1568-71.

18. Stoner SC, Worrel JA, Jones MT, Farrar CA, Ramlatchman LV. Pharmacist-designed and -implemented pharmaceutical care plan for antipsychotic-induced movement disorders. Pharmacotherapy. 2000;20(5): 583-8.

19. Kalachnik JE, Sprague RL, Slaw KM. Training clinical personnel to assess for tardive dyskinesia. Prog Neuropsychopharmacol Biol Psychiatry. 1988; 12(5):749-62

20. Dixon L, Weiden P, Fraces A, Rapkin B. Management of neurolepticinduced movement disorders: effects of physician training. Am J Psychiatry. 1989;146(1):104-6.

21. Rating scales. In: Drug-induced movement disorders: a clinical guide to rating scales [Internet]. Lincoln (NE): College of Psychiatric and Neurologic Pharmacists; c2003-2014 [cited 2014 Dec 19]. Available from: www.cpnp.org/ed/movement-disorders\#scales

22. Stoner S, Kalachnik J, Sommi R, Kane J. Drug-induced movement disorders: a clinical guide to rating scales [DVD]. Lincoln (NE): College of Psychiatric and Neurologic Pharmacists; 2012.

Alessandra Spadaro, RPh, BScPhm, ACPR, is with the Centre for Addiction and Mental Health, Toronto, Ontario.

Jamie Kellar, RPh, BScPhm, PharmD, is with the Centre for Addiction and Mental Health, Toronto, Ontario.

Gary Remington, $\mathrm{PhD}, \mathrm{MD}, \mathrm{FRCPC}$, is with the Centre for Addiction and Mental Health, Toronto, Ontario.

Beth Sproule, RPh, BScPhm, PharmD, is with the Centre for Addiction and Mental Health, Toronto, Ontario.

Mayce Al-Sukhni, RPh, BScPhm, PharmD, is with the Centre for Addiction and Mental Health, Toronto, Ontario.

Albert Chaiet, RPh, BScPhm, MScPhm, MBA, is with the Centre for Addiction and Mental Health, Toronto, Ontario.

Competing interests: For activities outside the submitted work, Gary Remington reports grants for a phase I trial and personal consultant fees from Neurocrine Biosciences, grants from Medicure for a single-site drug trial, personal consultant fees from Laboratorios Farmacéuticos Rovi and Synchroneuron, and speaker's honoraria from Novartis. No other competing interests were declared.

Address correspondence to:

Alessandra Spadaro

Centre for Addiction and Mental Health

1001 Queen Street W

Toronto ON M6J $1 \mathrm{H} 4$

e-mail: sandra.spadaro@camh.ca 